\title{
Lactobacillus gasseri LA39 Activates the Oxidative Phosphorylation Pathway in Porcine Intestinal Epithelial Cells
}

OPEN ACCESS

Edited by:

Djamel Drider,

Lille University of Science

and Technology, France

Reviewed by:

Alex Galanis,

Democritus University of Thrace,

Greece

Daniela Fiocco,

University of Foggia, Italy

*Correspondence:

Xianghua Yan

xhyan@mail.hzau.edu.cn

${ }^{t}$ These authors have contributed

equally to this work

Specialty section:

This article was submitted to

Food Microbiology,

a section of the journal

Frontiers in Microbiology

Received: 05 September 2018

Accepted: 22 November 2018

Published: 11 December 2018

Citation:

Hu J, Ma L, Zheng W, Nie Y and Yan X (2018) Lactobacillus gasseri

LA39 Activates the Oxidative Phosphorylation Pathway in Porcine

Intestinal Epithelial Cells.

Front. Microbiol. 9:3025.

doi: 10.3389/fmicb.2018.03025

\author{
Jun $\mathrm{Hu}^{1,2,3+}$, Libao $\mathrm{Ma}^{1,2,3 t}$, Wenyong Zheng ${ }^{1,2,3}$, Yangfan $\mathrm{Nie}^{1,2,3}$ and Xianghua Yan ${ }^{1,2,3 *}$ \\ 'State Key Laboratory of Agricultural Microbiology, College of Animal Sciences and Technology, Huazhong Agricultural \\ University, Hubei, China, ${ }^{2}$ The Cooperative Innovation Center for Sustainable Pig Production, Hubei, China, ${ }^{3}$ Hubei \\ Provincial Engineering Laboratory for Pig Precision Feeding and Feed Safety Technology, Hubei, China
}

Intestinal microbial interactions with the host epithelium have important roles in host health. Our previous data have suggested that Lactobacillus gasseri LA39 is the predominant intestinal Lactobacillus in weaned piglets. However, the regulatory role of L. gasseri LA39 in the intestinal epithelial protein expression in piglets remains unclear. In the present study, we conducted comparative proteomics approach to investigate the intestinal epithelial protein profile alteration caused by L. gasseri LA39 in piglets. The expressions of 15 proteins significantly increased, whereas the expressions of 13 proteins significantly decreased in the IPEC-J2 cells upon L. gasseri LA39 treatment. Bioinformatics analyses, including COG function annotation, GO annotation, and KEGG pathway analysis for the differentially expressed proteins revealed that the oxidative phosphorylation (OXPHOS) pathway in IPEC-J2 cells was significantly activated by L. gasseri LA39 treatment. Further data indicated that two differentially expressed proteins UQCRC2 and TCIRG1, associated with the OXPHOS pathway, and cellular ATP levels in IPEC-J2 cells were significantly up-regulated by L. gasseri LA39 treatment. Importantly, the in vivo data indicated that oral gavage of $L$. gasseri LA39 significantly increased the expression of UQCRC2 and TCIRG1 and the cellular ATP levels in the intestinal epithelial cells of weaned piglets. Our results, both in vitro and in vivo, reveal that $L$. gasseri LA39 activates the OXPHOS pathway and increases the energy production in porcine intestinal epithelial cells. These findings suggest that $L$. gasseri LA39 may be a potential probiotics candidate for intestinal energy production promotion and confers health-promoting functions in mammals.

Keywords: Lactobacillus gasseri LA39, intestinal epithelium, oxidative phosphorylation, iTRAQ, piglets

\section{INTRODUCTION}

Growing evidence has suggested that intestinal microbes have critical roles in intestinal homeostasis and host health (Sommer and Backhed, 2013). Intestinal microbes have been found to function in host immune defense system maturation (Ivanov et al., 2009), intestinal epithelium differentiation (Sommer and Backhed, 2013), and nutrients digestion (Backhed et al., 2007). However, 
intestinal microbial dysbiosis can cause host gastrointestinal diseases (Borody and Khoruts, 2011), such as irritable bowel syndrome, inflammatory bowel disease, and diarrhea. An increasing number of studies have investigated the healthpromoting roles of probiotics (Ventura et al., 2009; Lemon et al., 2012). According to the Food and Agriculture Organization of the United Nations and the WHO (FAO/WHO), probiotics are defined as "live microorganisms which when administered in adequate amounts confer a health benefit on the host" (Hill et al., 2014). The intestinal microbe-host interaction has become a research focus in microbiology (Kim et al., 2010). Thus, exploring the potential regulatory role of probiotic candidates in intestinal epithelium is of great significance.

Proteomics has been used in intestinal microbiota-host interaction research (Olivares et al., 2012; Siciliano and Mazzeo, 2012; van de Guchte et al., 2012; Luo et al., 2013; Ayllón et al., 2017). Olivares et al., using 2-dimensional gel electrophoresis (2-DE) and MALDI-TOF-TOF peptide fingerprinting, showed that oral gavage of rats with Bifidobacterium longum modulated the jejunal proteome in rats (Olivares et al., 2012). Luo et al. demonstrated that the probiotic, Enterococcus faecium altered the proteome in the intestinal mucosa of the broilers. Using 2-D fluorescence difference gel electrophoresis (DIGE), they exhibited that the chickens fed with $E$. faecium used less nutrient and energy while responding to the immune and antioxidant stresses (Luo et al., 2013). Ayllón et al., 2017 suggested that both host and pathogen factors were responsible for the commensal or infectious character of Campylobacter jejuni in different hosts, using sequential window acquisition of all theoretical fragment ion-mass spectrometry (SWATH-MS). Currently, a proteomics approach, termed isobaric tags for relative and absolute quantification (iTRAQ), has been widely performed to evaluate the alteration of protein expression profiles because of its several advantages, including high sensitivity, high-throughput, and accuracy (Treumann and Thiede, 2010). It has been used by us to investigate the alteration of protein expression profiles in weaned piglets (Fan et al., 2017; Hu et al., 2017). However, the iTRAQ strategy has not been used to evaluate the role of intestinal microbes in intestinal epithelial protein expression regulation.

Given the similarities in the anatomy and nutritional physiology between pigs (Sus scrofa) and human beings, the pig has been used as a research model (Vodicka et al., 2005; Maxmen, 2012; Meurens et al., 2012; Nielsen et al., 2014). Considering that members of the Lactobacillus genus may be potential probiotic candidates (Kleerebezem et al., 2010; van Baarlen et al., 2013), we mainly focused on the healthpromoting roles of a Lactobacillus species. Our previous data showed that Lactobacillus gasseri LA39 was a predominant intestinal Lactobacillus in the weaned piglets ( $\mathrm{Hu}$ et al., 2016). L. gasseri LA39 was found to produce gassericin A bacteriocin (Kawai et al., 1994) which is active against several pathogenic bacteria (Kawai et al., 2001), suggesting a probiotic potential of L. gasseri LA39. However, the regulatory role of L. gasseri LA39 in the expression of the intestinal epithelial proteins remains unclear. The present study was designed to investigate the potential role of L. gasseri LA39 in intestinal epithelium regulation using iTRAQ strategy. Our findings revealed that L. gasseri LA39 could activate the oxidative phosphorylation (OXPHOS) pathway in porcine intestinal epithelial cells showed. The ATP levels were also significantly increased with L. gasseri LA39 treatment. Our results suggest that L. gasseri LA39 may facilitate increasing intestinal energy production and confer health-promoting functions in mammals. Our data also further suggest the important interactions of gut microbes with host physiology in mammals.

\section{MATERIALS AND METHODS}

\section{In vitro Assay of Bacterial Adhesion to Intestinal Porcine Epithelial Cell Line From the Jejunum (IPEC-J2) Cells}

IPEC-J2 cells were cultured in DMEM/F12 (Gibco, 11320-033) containing $10 \%$ FBS (Gibco, 10099-141) in $5 \% \mathrm{CO}_{2}$ at $37^{\circ} \mathrm{C}$. The L. gasseri LA39 (JCM 11657) was obtained from the Japan Collection of Microorganisms (JCM). It was cultured in de Man, Rogosa and Sharpe (MRS) medium at $37^{\circ} \mathrm{C}$ in an anaerobic incubator. The viable IPEC-J2 cell count was performed by trypan blue staining and subsequent microscopic counting, and the viable L. gasseri LA39 count was performed with methylene blue staining, followed by microscopic counting. To investigate the bacterial adhesion to IPEC-J2 cells, we used a method combining bacterial fluorescein-5-isothiocyanate (FITC) labeling and intestinal epithelial cellular plasma membrane labeling by wheat germ agglutinin (WGA). Briefly, viable L. gasseri LA39 were co-incubated with FITC dye (Thermo Fisher Scientific, F1906) for $1 \mathrm{~h}$ at room temperature, and then washed 3 times with PBS. Subsequently, FITC-labeled L. gasseri LA39 were added into the IPEC-J2 cell medium for $2 \mathrm{~h}$ or $4 \mathrm{~h}$, the initial ratios of L. gasseri LA39 numbers to IPEC-J2 cells numbers were $1: 1,10: 1$, or 100:1. Following co-culture, IPECJ2 cells were washed three times with PBS to remove the L. gasseri LA39 that has not adhered and then fixed with $4 \%$ paraformaldehyde solution. After washing three times with PBS buffer, the IPEC-J2 cells were incubated with $5 \mu \mathrm{g} / \mathrm{ml}$ WGA (Thermo Fisher Scientific, W32466) at room temperature for $10 \mathrm{~min}$. Finally, FITC fluorescence (excitation $488 \mathrm{~nm}$ ) and WGA fluorescence (excitation $633 \mathrm{~nm}$ ) were detected by a laser scanning confocal microscope (ZEISS, LSM 880). The IPEC-J2 cells stained with WGA were shown by red color and the L. gasseri LA39 (adhered) stained with FITC dye were shown by green color in the fluorescence images. The numbers of adhered bacterial cells were calculated using the ratios of L. gasseri LA39 numbers to IPEC-J2 cells numbers in the fluorescence images. The final relative numbers of adhered bacterial cells (fold change) were normalized to the numbers of adhered bacterial cells (FITC-labeled L. gasseri LA39 were added into IPEC-J2 cell medium for $2 \mathrm{~h}$, and the initial ratio of L. gasseri LA39 numbers to IPEC-J2 cells numbers was $1: 1)$. 


\section{Co-culture Assay of IPEC-J2 Cells and L. gasseri LA39}

The L. gasseri LA39 used in the present study is a facultative anaerobe and can also survive under aerobic conditions, thereby such an in vitro model is appropriate to investigate the intestinal epithelial responses to L. gasseri LA39. Considering that the numbers of adhered bacterial cells were the largest when the co-culture assay was conducted for $4 \mathrm{~h}$ and the initial ratio of bacterial cells numbers to IPEC-J2 cells numbers was 100:1 in our data, we chose this co-culture protocol (including the co-culture time and initial ratios for interaction) to investigate the intestinal epithelial cellular responses to L. gasseri LA39. The IPEC-J2 cells and the viable $L$. gasseri $L A 39$ count was performed by staining as mentioned previously. Subsequently, viable L. gasseri LA39 were added into the IPEC-J2 cell medium for $4 \mathrm{~h}$, the initial ratio of L. gasseri LA39 numbers to IPEC-J2 cells numbers was 100:1. Similar dilutions of DMEM/F12 medium were used as vehicle controls.

\section{Protein Extraction, Trypsin Digestion, and ITRAQ Labeling}

Upon completion of the co-culture assay, the IPEC-J2 cells were washed three times with sterile PBS buffer to remove the L. gasseri LA39. The IPEC-J2 cells were frozen with liquid nitrogen immediately and then lysed in the "lysis buffer (8 M Urea, $40 \mathrm{mM}$ Tris- $\mathrm{HCl}$ with $1 \mathrm{mM}$ PMSF, $2 \mathrm{mM}$ EDTA, and 10 mM DTT, pH 8.5)" (Li et al., 2018). "After centrifugation at $25,000 \mathrm{~g}$ at $4^{\circ} \mathrm{C}$ for $20 \mathrm{~min}$, the supernatant obtained was reduced with $10 \mathrm{mM} \mathrm{DTT}$ at $56^{\circ} \mathrm{C}$ for $1 \mathrm{~h}$, and then alkylated with $55 \mathrm{mM}$ iodoacetamide (IAM) in the dark at room temperature for $45 \mathrm{~min}$ " (Huang et al., 2018). Following further centrifugation with $25,000 \mathrm{~g}$ at $4^{\circ} \mathrm{C}$, the supernatant was quantified by Bradford method. The protein solution $(100 \mu \mathrm{g})$ was digested using Trypsin Gold. Following trypsin digestion, "the peptides were desalted, vacuum-dried, and dissolved in $30 \mu \mathrm{l}$ of $0.5 \mathrm{M} \mathrm{TEAB}$ and labeled by the iTRAQ Reagent 8-plex Kit. The peptides labeled with different reagents were combined, and then desalted and vacuum-dried" (Li et al., 2018).

\section{Peptide Fractionation, High Performance Liquid Chromatography (HPLC), and Mass Spectrometer Detection}

The peptides fractionation, high performance liquid chromatography (HPLC), and mass spectrometer detection were performed using the previously described procedures (Wang et al., 2018).

\section{Bioinformatics Analyses}

The raw MS/MS data was converted into the MGF format using the Proteome Discoverer. The peptide spectrum from raw data will be pre-processed to obtain the peptide spectrum of high quality by filtering out these peptide spectrums (ionic charge > 7). "Proteins were identified by the Mascot search engine" (Weatherly et al., 2005) run against the Uniprot database for Sus scrofa. "At least one unique peptide was essential for the identified protein." The parameters are shown in the Table 1. "An automated software, named IQuant, was conducted to analyze the labeled peptides with isobaric tags quantitatively" (Wen et al., 2014). The main IQuant quantitation parameters are shown in Table 2. False discovery rate (FDR) was used to control the confidence of peptides and proteins as previously described (Savitski et al., 2015; Hu Z. et al., 2018).

Based on some previous iTRAQ analyses (Rhein et al., 2009; Ren et al., 2013; Huang et al., 2015; Shi et al., 2015; Hu et al., 2017), "a ratio of 1.2 -fold ( $>1.20$ or $<0.833$ ) with a $q$-value $<0.05$ were chosen as the cutoff for up-regulated or down-regulated expression, respectively." To better detect the differentially expressed proteins, "we further defined those proteins significantly altered in at least two replicates as differentially expressed proteins as previously described" (Zhu et al., 2009; Long et al., 2016). "Functional annotations of identified proteins were conducted by COG and GO, respectively." "We performed a hyper-geometric test to obtain target GO terms in the GO enrichment analysis using our previously described procedures" (Hu et al., 2017). "KEGG enrichment analysis was also performed to identify the KEGG pathways preferentially affected by treatment using our previously described procedures" (Hu et al., 2017).

\section{In vivo Assay for Piglets and Sample Collection}

Lactobacillus gasseri LA39 was cultured in MRS medium at $37^{\circ} \mathrm{C}$. 100 crossbred piglets (Landrace $\times$ Yorkshire) with similar birth weight were randomly divided into two groups. Piglets in the same group were randomly divided into 5 pens, and 10 piglets per pen. The piglets in control (Ctrl) group were oral administrated with a vehicle (sterile PBS, $2 \mathrm{ml}$ ) once a day from the age of 6-20 days. The piglets in L. gasseri LA39 (LG) group were oral

TABLE 1 | Mascot search parameters.

\begin{tabular}{ll}
\hline Item & Value \\
\hline Type of search & MS/MS ion search \\
Enzyme & Trypsin \\
Fragment mass tolerance & $0.05 \mathrm{Da}$ \\
Mass values & Monoisotopic \\
Variable modification & Oxidation (M), iTRAQ8plex (Y) \\
Peptide mass Tolerance & 20 ppm \\
Fixed modification & Carbamidomethyl (C), iTRAQ8plex (N-term), \\
& iTRAQ8plex (K) \\
Database & Uniprot database for Sus scrofa
\end{tabular}

TABLE 2 | IQuant quantitation parameters.

\begin{tabular}{ll}
\hline Item & Value \\
\hline Quant peptide & Use All unique peptide \\
Quant number & At least one unique spetra \\
Normalization & VSN \\
Protein Ratio & Weighted average \\
Statistical Analysis & Permutation Tests
\end{tabular}


administrated with a bacterial suspension $\left(2 \mathrm{ml}, 10^{8} \mathrm{CFU} / \mathrm{ml}\right.$ L. gasseri LA39 in PBS) once a day from the age of 6-20 days. All the piglets were early-weaned at 21 days of age. Each piglet was randomly chosen from each pen at 26 days of age and these piglets selected from Ctrl group (5 piglets) and LG group (5 piglets) were slaughtered. The intestinal tissues were collected from the approximately middle positions in intestinal tracts, including duodenum, jejunum, and ileum, respectively, to reduce sample variability as previously described (Hu et al., 2017). Piglets handling protocols were approved by the Institutional Animal Care and Use Committee of Huazhong Agricultural University. The methods were carried out in accordance with the approved guidelines.

\section{Western Blotting}

The whole cell lysates (WCLs) of IPEC-J2 cells and the WCLs of intestinal epithelial cells from weaned piglets used for western blotting were prepared using RIPA lysis buffer (Sangon Biotech, C500005). Western blotting was conducted by our previously described methods (Hu et al., 2017). Below are the antibodies used in the western blotting: horseradish peroxidase (HRP)conjugated secondary goat anti-rabbit antibodies (Santa Cruz Biotechnology, sc-2004), UQCRC2 antibody (Abcam, ab203832), TCIRG1 antibody (Proteintech, 12649-1-AP), and GAPDH (ABclone Technology, AC001).

\section{Measurement of Relative mRNA Expression of Proteins}

Total RNA was extracted from the IPEC-J2 cells and intestinal epithelial cells of weaned piglets. Subsequently, cDNA was generated by the PrimeScript TM RT reagent Kit (Takara, RR047A). The relative mRNA expression of these proteins (UQCRC2, TCIRG1, and GAPDH) was measured using qRTPCR. The specific primers used in qRT-PCR were shown in Table 3. qRT-PCR was conducted using our previously described procedures (Hu J. et al., 2018).

\section{Measurement of Cellular ATP Levels}

The total cellular ATP contents in IPEC-J2 cells and intestinal epithelial cells (including duodenum, jejunum, and ileum) from weaned piglets were measured by an ATP determination kit (Thermo Scientific, A22066) according to the manufacturer's protocol. The protein concentration of the WCLs was measured by the protein assay kit (Thermo Scientific, 23227). The ATP

TABLE 3 | Specific primers for UQCRC2, TCIRG1, and GAPDH genes.

\begin{tabular}{lll}
\hline Gene name & & Primers sequences \\
\hline UQCRC2 & Forward & 5'-AGCCATCCACGGTCCTTCA-3' \\
& Reverse & 5'-GCTTGCTGCCATTGACTTCC-3' \\
TCIRG1 & Forward & 5'-ACGCTTTGGGTGGATGTTC-3' \\
& Reverse & 5'-CAGGCGATCCGACTCTTCTT-3' \\
GAPDH & Forward & 5'-CCTTCATTGACCTCCACTACAT-3' \\
& Reverse & $5^{\prime}$-GGATCTCGCTCCTGGAAGA-3'
\end{tabular}

concentration $(\mathrm{nmol} / \mathrm{L})$ was normalized to the total protein concentration $(\mathrm{mg} / \mathrm{L})$ of WCLs.

\section{Statistical Analyses}

Statistical analyses were conducted by GraphPad Prism (version 6.0c) software. Two-way analysis of variance (ANOVA) and adjustments for multiple comparisons was conducted for Figure 1B. The Student's $t$-test was conducted to compare the differences between the two groups in Figures 5C-F, 6. The data are shown as mean \pm SEM (ns, not significant, ${ }^{*} p<0.05$, ** $p<0.01)$.

\section{RESULTS}

\section{Identification of the Differentially Expressed Proteins in IPEC-J2 Cells by Co-culturing With L. gasseri LA39}

To investigate the effects of L. gasseri LA39 on the porcine intestinal epithelial cells, we used a co-culture method by which live L. gasseri LA39 were added into the IPEC-J2 cells medium. The results of the adherence assay indicated that the numbers of adhered bacterial cells were the largest when the co-culture was conducted for $4 \mathrm{~h}$ and the initial ratio of bacterial cell numbers to IPEC-J2 cell numbers was 100:1 (Figures 1A,B). Thus, we used the iTRAQ strategy, where live L. gasseri LA39 were added into the IPEC-J2 cell medium for $4 \mathrm{~h}$ at 100:1 ratio to compare the intestinal epithelial cell protein profiles between the L. gasseri LA39 (LG) group and the control (Ctrl) group in the co-culture assay. The workflow for the iTRAQ is shown in Figure 1C. In total, three biological replicates of IPEC-J2 cells from LG and Ctrl groups were used in this study. The tryptic peptides were labeled with isobaric iTRAQ tags (LG: 115; Ctrl: 113), and these labeled peptides were detected using liquid chromatographyelectrospray ionization-tandem mass spectrometry (LC-ESIMS/MS) method.

Our data from iTRAQ indicated that a total of 6148,6132 , and 6161 proteins were quantified in the three biological replicates, respectively (Figure 2A). Of these quantified proteins, 4841 proteins were commonly identified in all the three biological replicates (Figure 2A). Based on those previous iTRAQ studies (Rhein et al., 2009; Ren et al., 2013; Huang et al., 2015; Shi et al., 2015; Hu et al., 2017), an iTRAQ ratio of 1.2 -fold $(>1.20$ or $<0.833$ ) with a $q$-value $<0.05$ was chosen as the cutoff for up-regulated or down-regulated expression, respectively. To better detect the differentially expressed proteins, we further defined these proteins significantly altered in at least two replicates as the differentially expressed proteins as previously described (Zhu et al., 2009; Long et al., 2016). As shown in the scatter plot and heat map, the expressions of 15 proteins significantly increased, whereas the expressions of 13 proteins significantly decreased in IPEC-J2 cells after L. gasseri LA39 treatment (Figures 2B,C). The detailed information for differentially expressed proteins is listed in Table 4. 
A

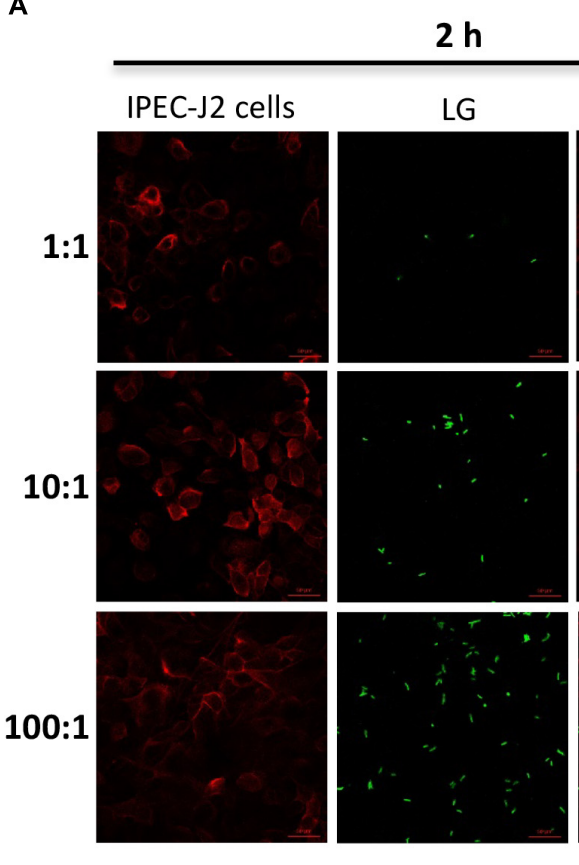

B

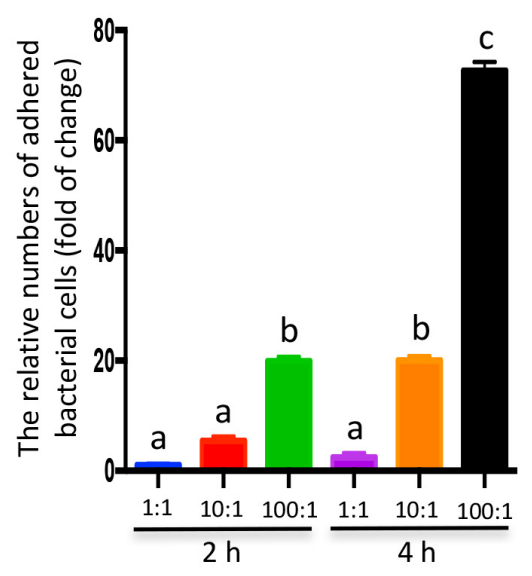

$4 \mathrm{~h}$

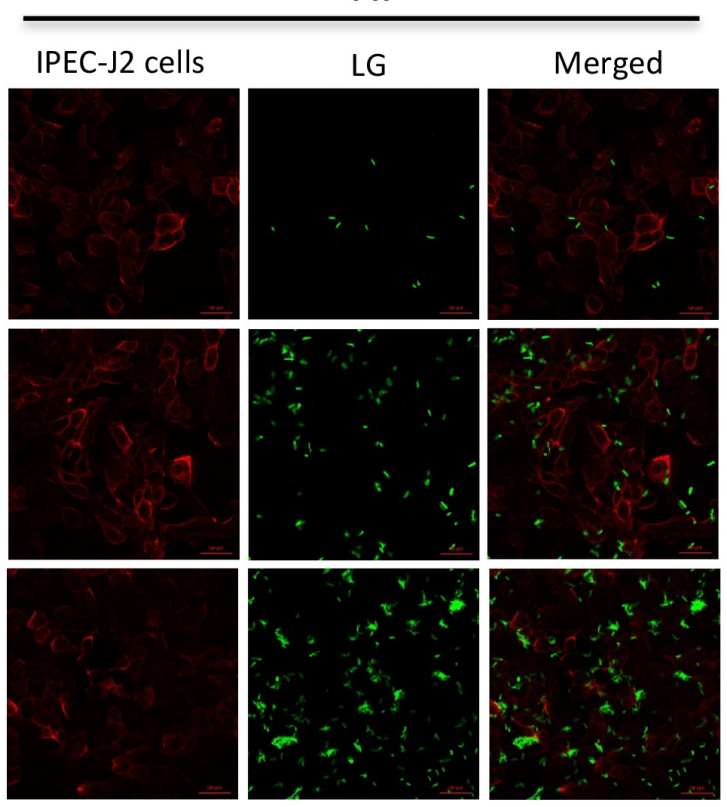

C

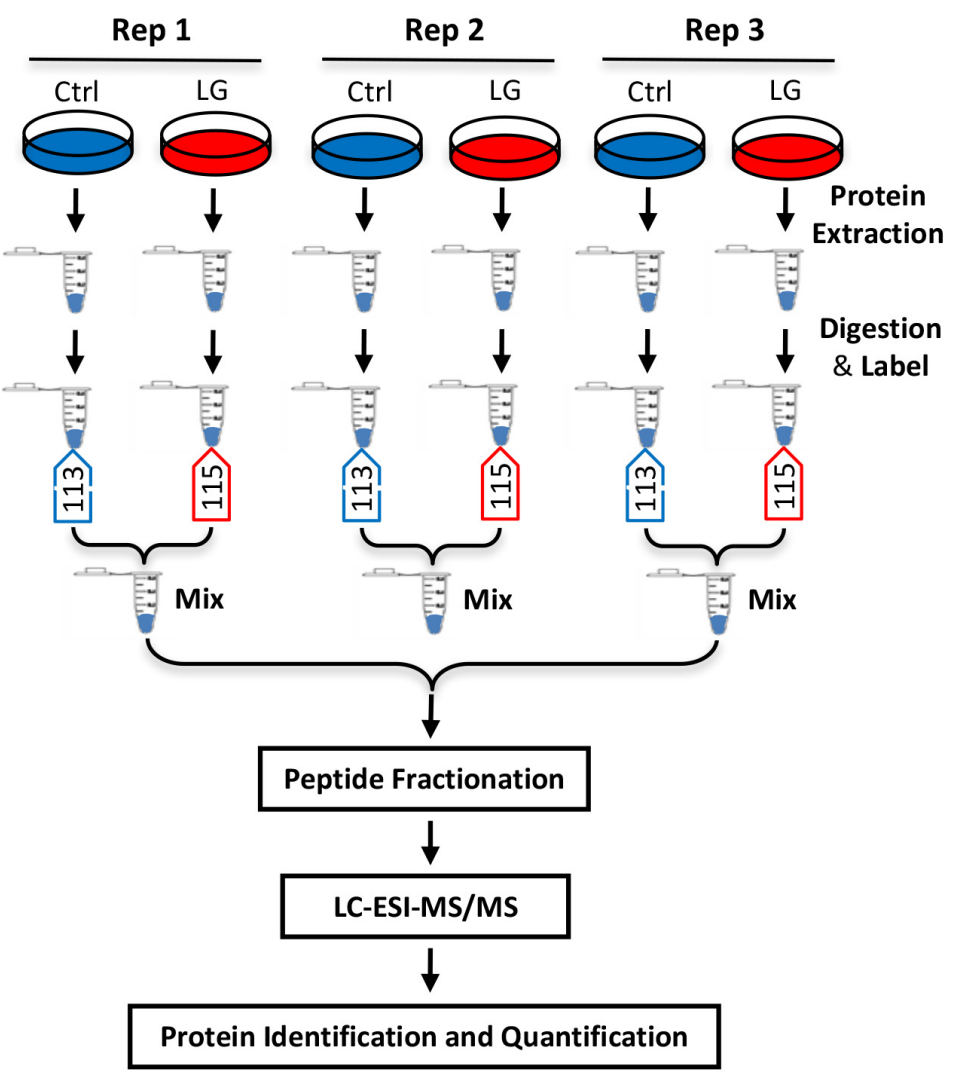

FIGURE 1 | Experimental design and schematic workflow for iTRAQ assay. (A) In vitro assay of bacterial adhesion to IPEC-J2 cells was shown by fluorescence staining. FITC-labeled L. gasseri LA39 (green) were added into IPEC-J2 cell medium for 2 or $4 \mathrm{~h}$ and the initial ratios of L. gasseri LA39 numbers to IPEC-J2 cell numbers were 1:1, 10:1, or 100:1. The plasma membranes of IPEC-J2 cells were labeled by wheat germ agglutinin (WGA) dye (red). (B) The relative numbers of adhered bacterial cells to IPEC-J2 cells. Data are represented as mean \pm SEM $(n=3)$. Different letters above the bars denotes a significant difference among the groups. (C) Schematic workflow for the iTRAQ assay as described in Materials and Methods. 
A

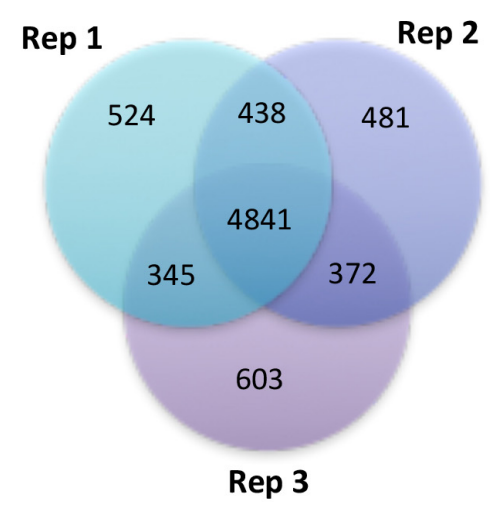

B

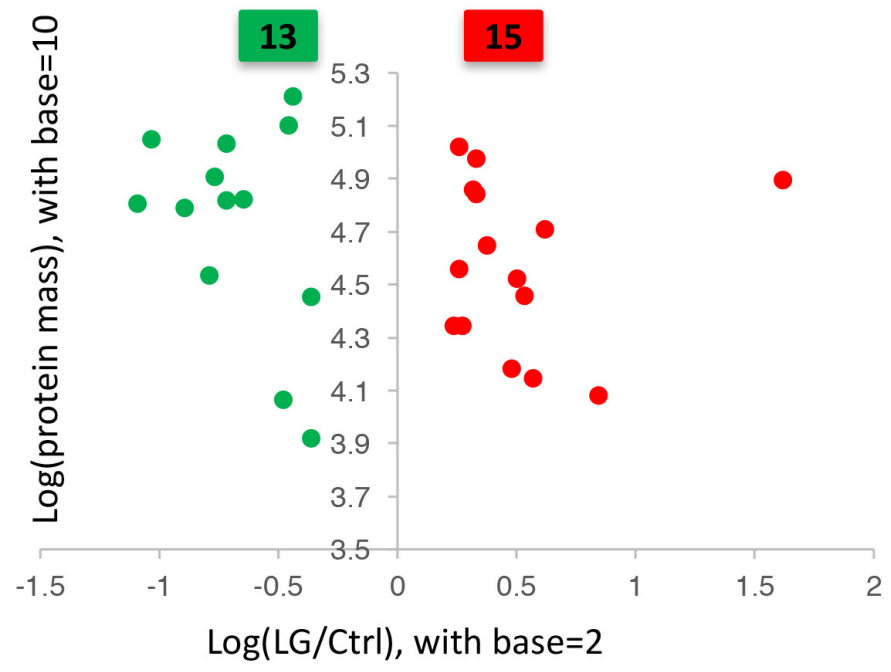

C
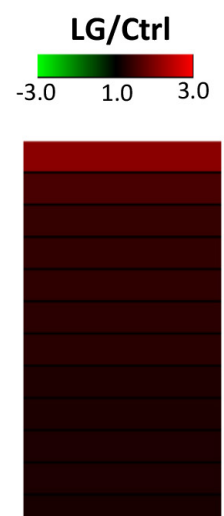

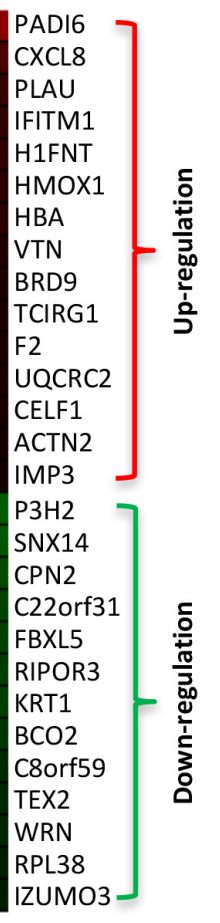

FIGURE 2 | Identification of differentially expressed proteins in IPEC-J2 cells upon L. gasseri LA39 treatment using iTRAQ strategy. (A) Venn diagrams for the quantified proteins in the three biological replicates. (B) Scatter plot analysis based on the differentially expressed proteins. The red spots and green spots indicate the significantly up-regulated proteins and significantly down-regulated proteins, respectively. (C) Heat map for the differentially expressed proteins. The values of the colors in the heat map indicate the mean ITRAQ ratio (fold change; LG/Ctrl) in protein expression.

\section{COG Annotation and Cellular Compartment Analyses of the Differentially Expressed Proteins}

To further uncover the effects of L. gasseri LA39 on the protein profiles of the IPEC-J2 cells, we used the COG annotation and GO annotation to analyze the differentially expressed proteins. The results showed that these COG functions, including energy production and conversion; post-translational modification, protein turnover, chaperones; translation, ribosomal structure and biogenesis were mainly annotated in the differentially expressed proteins (Figure 3A). Cellular compartment analysis from GO annotation indicated that the differentially expressed proteins were mainly located in the membrane, cytoplasm, plasma membrane, extracellular region, nucleolus, mitochondrion, intermediate filament cytoskeleton, endoplasmic reticulum, Golgi apparatus, and caveolae (Figure 3B). The subcellular localization in plasma membrane and extracellular region suggested a positive intestinal epithelial response to the extracellular L. gasseri LA39. The COG function "energy production and conversion" and the cellular compartment "mitochondrion" annotated in the differentially expressed proteins, suggested that L. gasseri LA39 treatment may have a regulatory role in the mitochondrial energy metabolism of porcine intestinal epithelial cells.

\section{Biological Process and Molecular Function Analyses of Differentially Expressed Proteins}

The GO terms for biological processes, including defense response, negative regulation of G-protein coupled receptor protein signaling pathway, regulation of response to external stimulus, regulation of G-protein coupled receptor protein 
TABLE 4 | Differentially expressed proteins quantified by iTRAQ strategy in IPEC-J2 cells.

\begin{tabular}{|c|c|c|c|c|c|c|}
\hline Accession & Gene name & Protein name & Ratio (Rep 1) & Ratio (Rep 2) & Ratio (Rep 3) & Ratio (mean) \\
\hline \multicolumn{7}{|c|}{ Up-regulated proteins (LG/ Ctrl) } \\
\hline K9J6L7 & TCIRG1 & V-type proton ATPase subunit a & $1.268^{*}$ & $1.274^{*}$ & $1.225^{*}$ & 1.26 \\
\hline P04185 & PLAU & Urokinase-type plasminogen activator & $1.553^{*}$ & & $1.519 *$ & 1.54 \\
\hline P32394 & HMOX1 & Heme oxygenase 1 & $1.380^{*}$ & $1.418^{*}$ & $1.457^{*}$ & 1.42 \\
\hline F1SUQ9 & PAD 16 & Peptidyl arginine deiminase 6 & & $2.889^{*}$ & $3.262^{*}$ & 3.08 \\
\hline F1RPD4 & UQCRC2 & Cytochrome b-c1 complex subunit 2 & 1.141 & $1.250^{*}$ & $1.242^{*}$ & 1.21 \\
\hline P01965 & HBA & Hemoglobin subunit alpha & $1.315^{*}$ & $1.320^{*}$ & $1.554^{*}$ & 1.4 \\
\hline F1RGC4 & IFITM1 & Interferon-induced transmembrane protein 1 & $1.720^{*}$ & $1.450^{*}$ & 1.286 & 1.49 \\
\hline Q19AZ8 & $\mathrm{F} 2$ & Prothrombin & $1.111^{*}$ & $1.417^{*}$ & $1.235^{*}$ & 1.25 \\
\hline F1SID9 & CELF1 & CUGBP Elav-like family member 1 & $1.202^{*}$ & 1.303* & $1.100^{*}$ & 1.2 \\
\hline I3L9F8 & VTN & Vitronectin & $1.159^{*}$ & $1.405^{*}$ & $1.323^{*}$ & 1.3 \\
\hline F1SGU6 & H1FNT & $\mathrm{H} 1$ histone family member $\mathrm{N}$, testis specific & 1.232 & $1.558^{*}$ & $1.556^{*}$ & 1.45 \\
\hline AOAOB8RW18 & BRD9 & Bromodomain containing 9 & 1.058 & $1.404^{*}$ & $1.330^{*}$ & 1.26 \\
\hline P26894 & CXCL8 & Interleukin-8 & $1.777^{*}$ & $1.680^{*}$ & $1.950^{*}$ & 1.8 \\
\hline F1SJ73 & IMP3 & U3 small nucleolar ribonucleoprotein & $1.267^{*}$ & 1.058 & $1.203^{*}$ & 1.18 \\
\hline F1RHL9 & ACTN2 & Alpha-actinin-2 & $1.235^{*}$ & $1.333^{*}$ & 1.018 & 1.2 \\
\hline \multicolumn{7}{|c|}{ Down-regulated proteins (LG/ Ctrl) } \\
\hline F1SGG3 & $\mathrm{KRT} 1$ & Keratin 1 & $0.588^{*}$ & $0.426^{*}$ & $0.818^{*}$ & 0.61 \\
\hline F1S5E2 & FBXL5 & F-box and leucine rich repeat protein 5 & & $0.519 *$ & $0.665^{*}$ & 0.59 \\
\hline F1SM94 & BC02 & Beta-carotene oxygenase 2 & & $0.677^{*}$ & $0.603^{*}$ & 0.64 \\
\hline I3LR87 & C22orf31 & Chromosome 22 open reading frame 31 & $0.573^{*}$ & $0.668^{*}$ & $0.507^{*}$ & 0.58 \\
\hline F1RSL6 & TEX2 & Testis expressed 2 & $0.683^{*}$ & $0.707^{*}$ & 0.800 & 0.73 \\
\hline F1RX70 & WRN & Werner syndrome RecQ like helicase & $0.795^{*}$ & $0.757^{*}$ & 0.656 & 0.74 \\
\hline I3LNH2 & RIPOR3 & RIPOR family member 3 & & $0.583^{*}$ & $0.635^{*}$ & 0.61 \\
\hline F1SP77 & IZUM03 & IZUMO family member 3 & 0.825 & $0.727^{*}$ & $0.802^{*}$ & 0.78 \\
\hline F2Z568 & RPL38 & Ribosomal protein L38 & $0.824^{*}$ & $0.763^{*}$ & $0.749 *$ & 0.78 \\
\hline I3LF89 & CPN2 & Carboxypeptidase $\mathrm{N}$ subunit 2 & $0.442^{*}$ & $0.528 *$ & $0.645^{*}$ & 0.54 \\
\hline F1SFH5 & $\mathrm{P} 3 \mathrm{H} 2$ & Prolyl 3-hydroxylase 2 & $0.505^{*}$ & & $0.440^{*}$ & 0.47 \\
\hline F1RXB9 & C8orf59 & Chromosome 8 open reading frame 59 & $0.706^{*}$ & $0.728^{*}$ & 0.725 & 0.72 \\
\hline $\mathrm{F} 1 \mathrm{SOH} 9$ & SNX14 & Sorting nexin 14 & $0.463^{*}$ & 0.576 & $0.419^{*}$ & 0.49 \\
\hline
\end{tabular}

* $q$-value $<0.05$

signaling pathway, and iron ion homeostasis were significantly enriched, further suggesting a positive intestinal epithelial response to extracellular L. gasseri LA39 stimulus (Figure 4A). Several GO terms for molecular functions, including endopeptidase activity, peptidase activity, iron ion binding, heme binding, oxidoreductase activity acting on paired donors with the incorporation or reduction of molecular oxygen, metal ion binding, and cation binding were significantly enriched, suggesting a potential role of L. gasseri LA39 in the mitochondrial respiratory chain of IPEC-J2 cells (Figure 4B).

\section{Oxidative Phosphorylation (OXPHOS) Pathway in IPEC-J2 Cells Is Activated by the L. gasseri LA39}

The KEGG pathway enrichment analysis of differentially expressed proteins was used to uncover the biological events in IPEC-J2 cells preferentially affected by L. gasseri LA39 treatment. The results indicated that several KEGG pathways, including complement and coagulation cascades, NF-kappa B signaling pathway, and OXPHOS were enriched (Figure 5A). Importantly, the expression levels of the differentially expressed proteins prothrombin, urokinase-type plasminogen activator, and vitronectin, involved in the complement and coagulation cascades pathway, significantly increased with L. gasseri LA39 treatment, suggesting the activation of the intestinal immune defense system. The expression levels of two differentially expressed proteins interleukin- 8 and urokinase-type plasminogen activator, involved in the NFkappa B signaling pathway, also significantly increased with L. gasseri LA39 treatment, indicating that the NF-kappa B signaling pathway in the intestinal epithelial cells was activated by L. gasseri LA39. Strikingly, the expression levels of two differentially expressed proteins UQCRC2 and TCIRG1, involved with the OXPHOS pathway, were significantly increased by L. gasseri LA39 treatment (Figure 5B). Combining the COG annotation and GO annotation analyses, the KEGG pathways analyses further suggested that the intestinal epithelial cellular mitochondrion functions, especially the OXPHOS pathway, might be preferentially affected by L. gasseri LA39 treatment. We further conducted the western blotting assay to validate the expression levels of the two differentially expressed proteins UQCRC2 and TCIRG1. The results demonstrated that the expression levels of UQCRC2 and TCIRG1 were significantly 
A

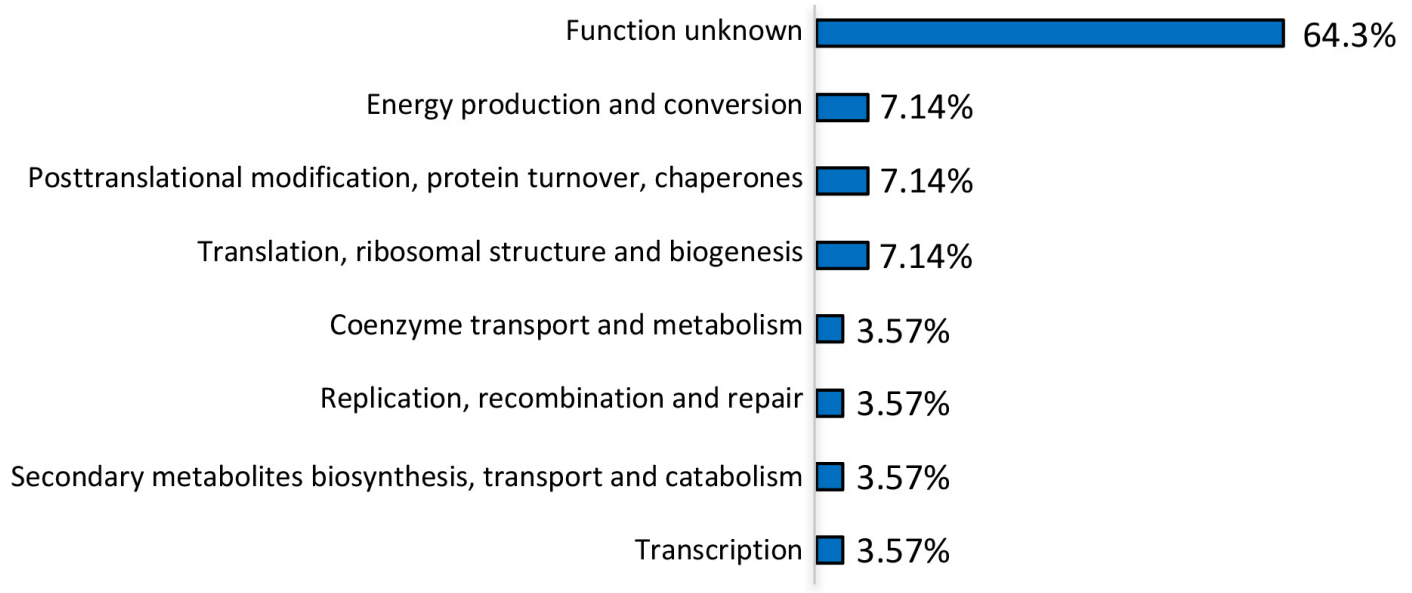

B

Cellular compartment analysis

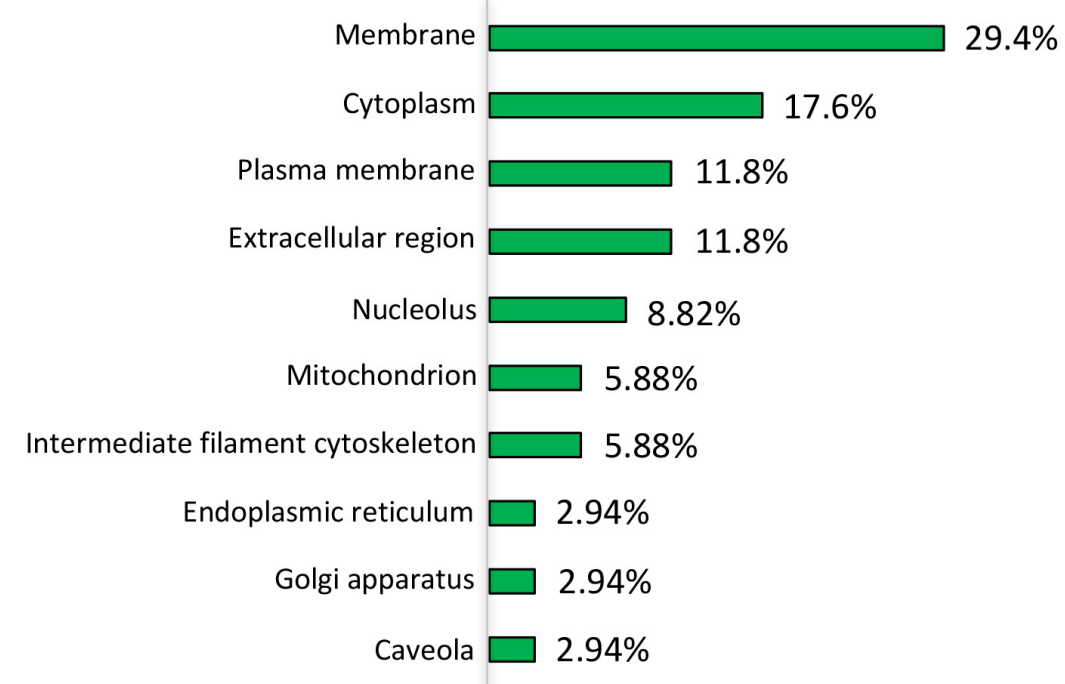

FIGURE 3 | COG function annotation and cellular compartment analyses for the differentially expressed proteins. (A) COG function annotation for the differentially expressed proteins. The relative proportions of the COG terms are shown on the right of the corresponding columns. (B) Cellular compartment analysis for the differentially expressed proteins. The relative proportions of the GO terms for cellular compartment are shown on the right of the corresponding columns.

up-regulated upon L. gasseri LA39 treatment, which was consistent with the results of the iTRAQ analysis (Figure 5C). Our data also showed that the relative mRNA expression levels of UQCRC2 and TCIRG1 were both significantly up-regulated with L. gasseri LA39 treatment (Figures 5D,E). Given that ATP, the major cellular energy source, is mainly produced by the OXPHOS, we then measured the levels of cellular ATP. The results demonstrated that the levels of ATP in IPEC-J2 cells were significantly increased by L. gasseri LA39 treatment (Figure 5F). These findings indicated that L. gasseri LA39 activates the OXPHOS pathway, resulting in an increase in the energy production in IPEC-J2 cells.

\section{Oral Administration of L. gasseri LA39 Activates the OXPHOS Pathway in Intestinal Epithelial Cells of Early-Weaned Piglets}

Based on the regulatory role of L. gasseri LA39 on the cultured IPEC-J2 cells, we next investigated the role of L. gasseri LA39 in the intestinal epithelial cells of weaned piglets in vivo. Our data indicated that oral gavage of L. gasseri LA39 significantly increased the expression levels and relative mRNA expression levels of UQCRC2 and TCIRG1 in the intestinal epithelial cells [including duodenum (Figures 6A-C), jejunum (Figures 6D-F), 
A

\section{Enrichments in biological process}

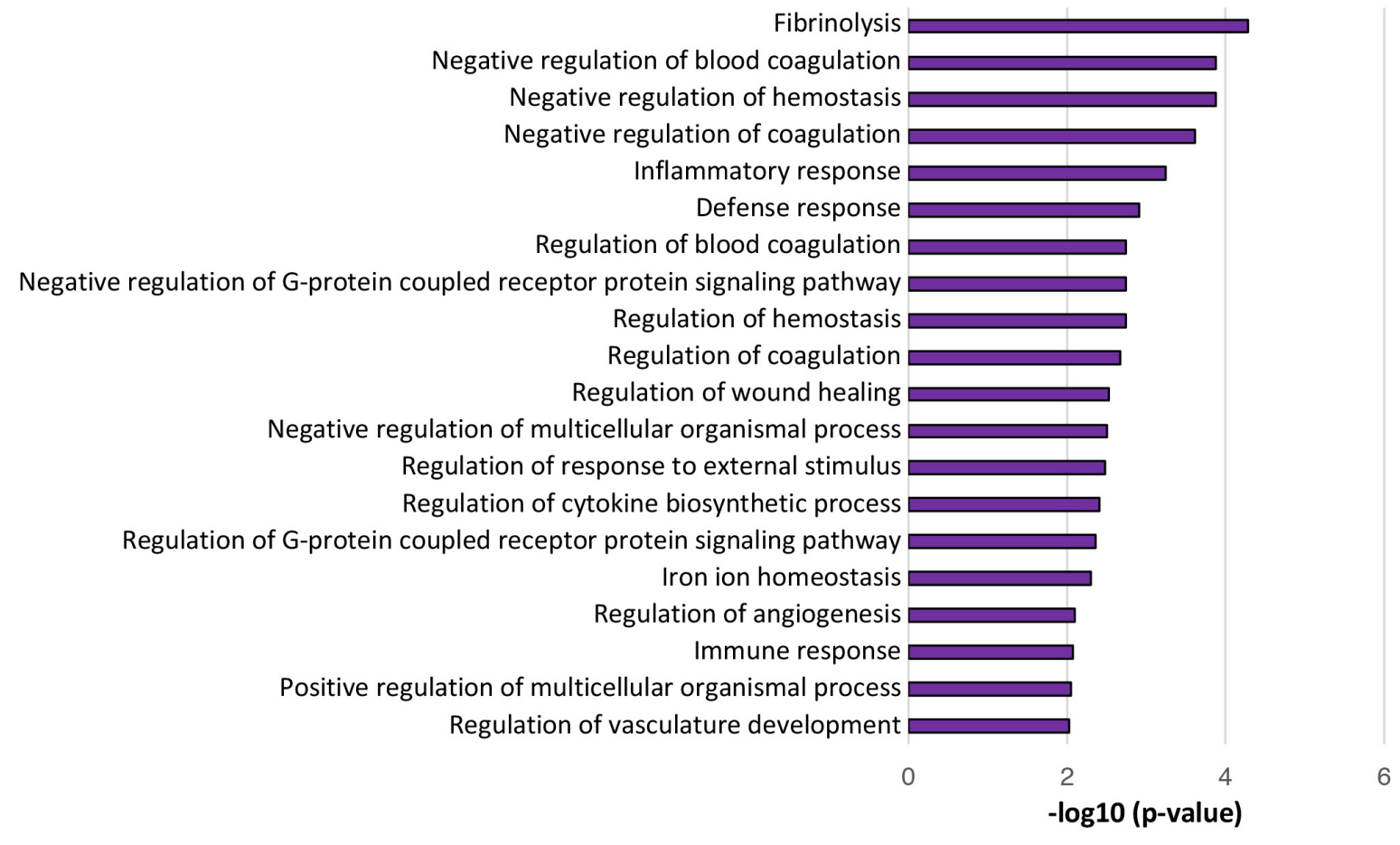

B

Enrichments in molecular function

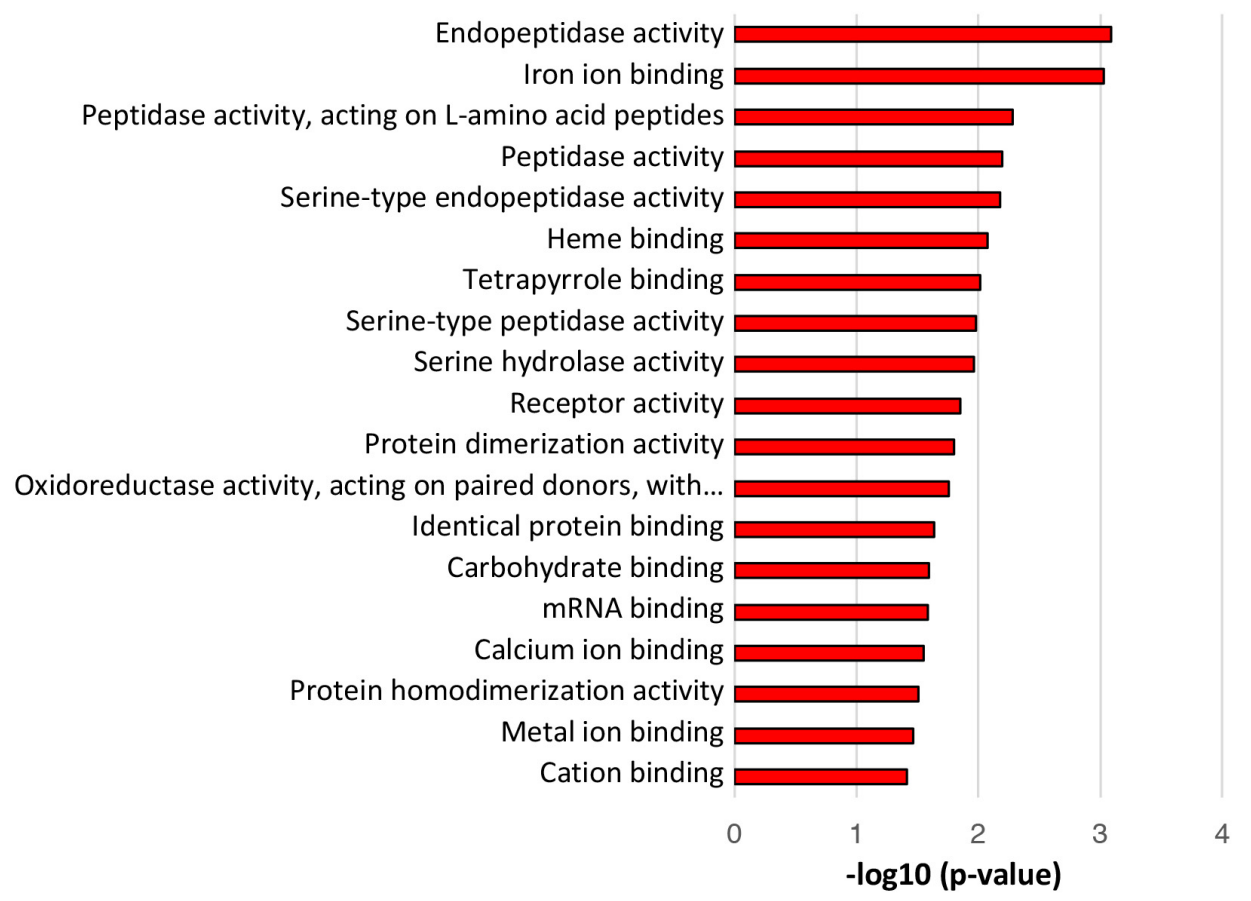

FIGURE 4 | Biological processes and molecular function enrichment analyses for the differentially expressed proteins. (A) The top twenty significantly enriched GO terms for biological processes. The values in the column represent the normalized $p$-values (-log10). The most enriched biological process is displayed at the top of the column. (B) The significantly enriched GO terms for molecular function. 
A

\section{Top ten enrichments in KEGG pathway}

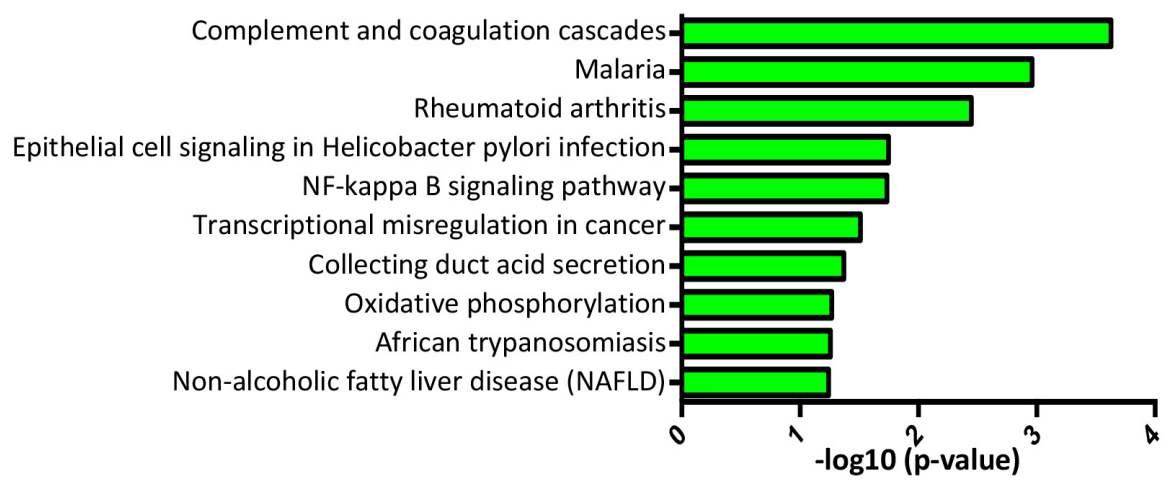

B

Oxidative phosphorylation

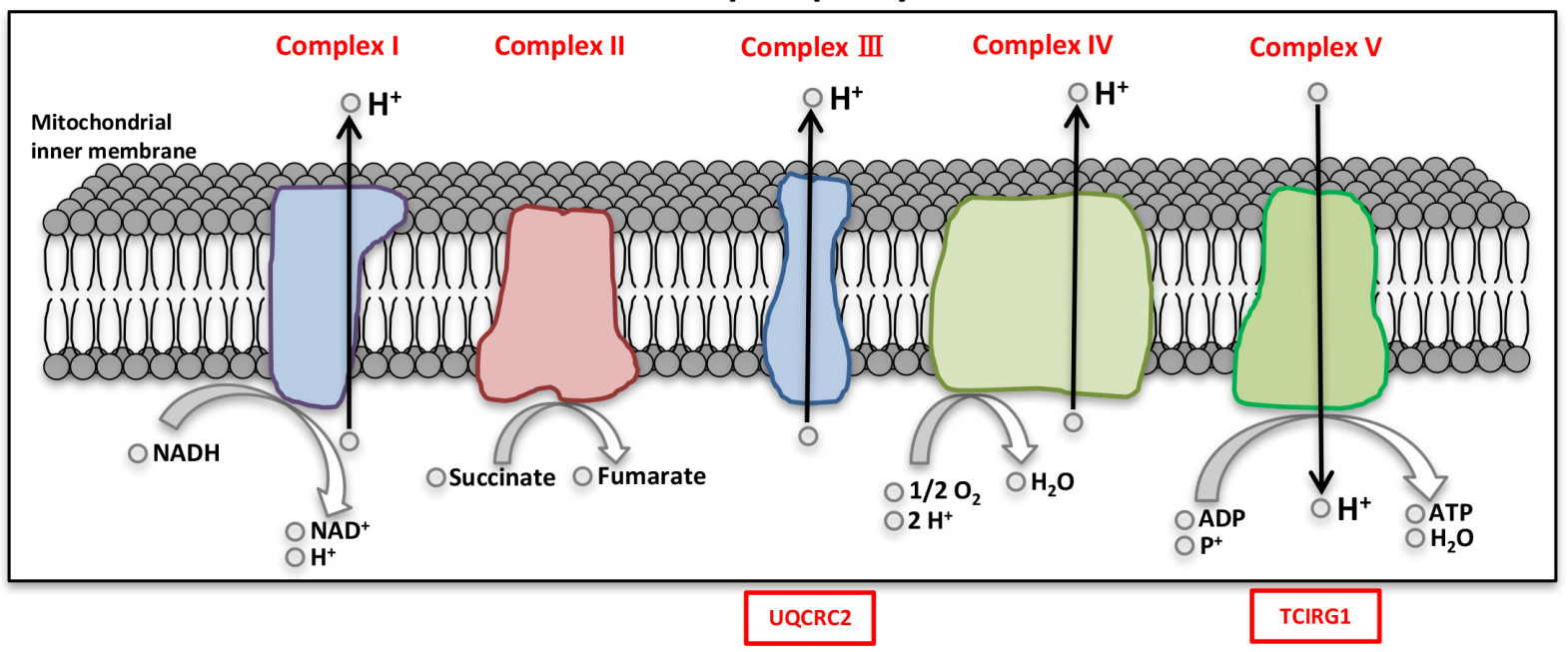

C
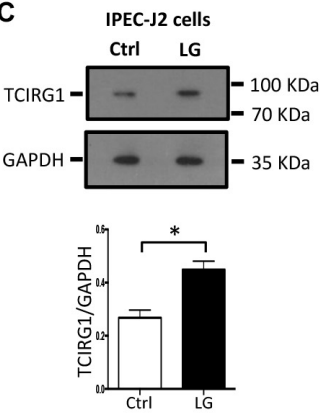

D

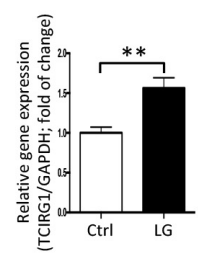

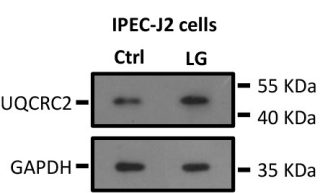

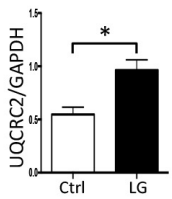

E

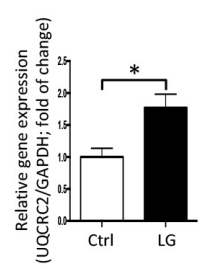

FIGURE 5 | The OXPHOS pathway in porcine intestinal epithelial cells is activated by L. gasseri LA39. (A) KEGG pathway enrichment analysis for differentially expressed proteins. Values in the column indicate the normalized $p$-values $(-\log 10)$. The most enriched KEGG pathway is displayed at the top of the column. 
FIGURE 5 | Continued

(B) Differential expression profiles of proteins involved in OXPHOS metabolic pathway. All differentially expressed proteins are shown below the corresponding complex in OXPHOS pathway map. Protein marked with a red box showed a significantly increased expression comparing the L. gasseri LA39 group with the Ctrl group. (C) Western blotting analysis of the expression levels of TCIRG1, UQCRC2, and GAPDH in IPEC-J2 cells. Normalization and quantitation of TCIRG1/GAPDH and UQCRC2/GAPDH are shown in the corresponding bar chart. (D,E) The relative mRNA expression of TCIRG1, UQCRC2, and GAPDH in IPEC-J2 cells. Normalization and quantitation of TCIRG1/GAPDH (D) and UQCRC2/GAPDH (E) were shown in corresponding bar chart. (F) ATP levels of IPEC-J2 cells. The ATP concentration $(\mathrm{nmol} / \mathrm{L})$ was normalized to the total protein concentration (mg/L) of WCLs. Data are shown as mean $\pm \mathrm{SEM} ; n=3(\mathrm{C}) ; n=5(\mathrm{E}) ; n=6(\mathrm{~F})$. ${ }^{*} p<0.01$, $* p<0.05$.

A

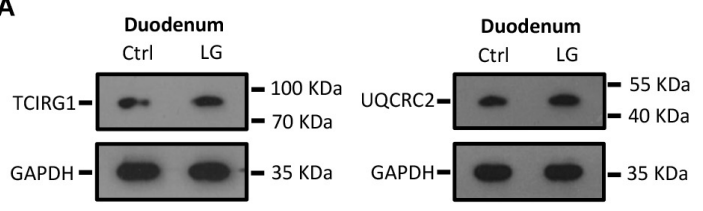

D

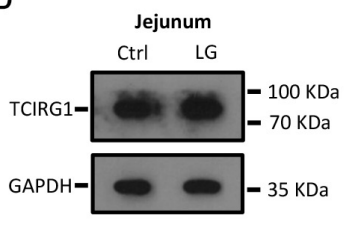

G

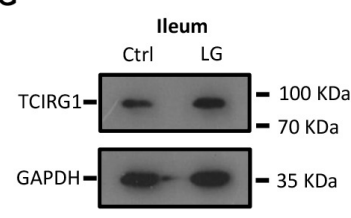

H

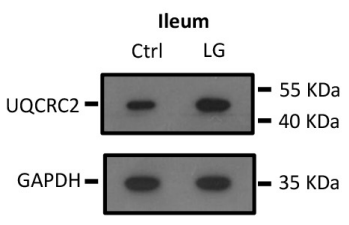

B

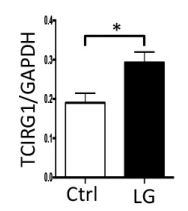

$\mathrm{E}$

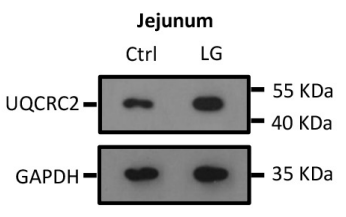

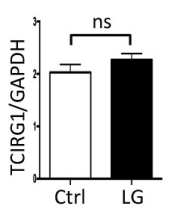

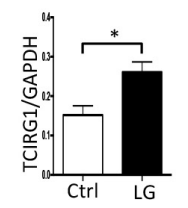

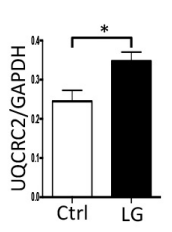

C

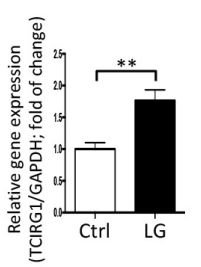

F

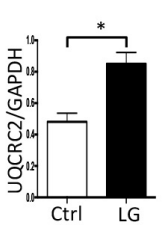

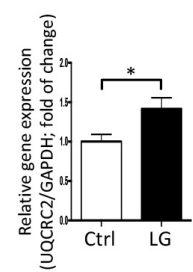

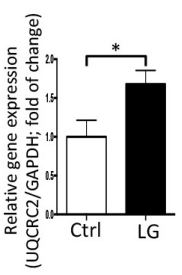

L
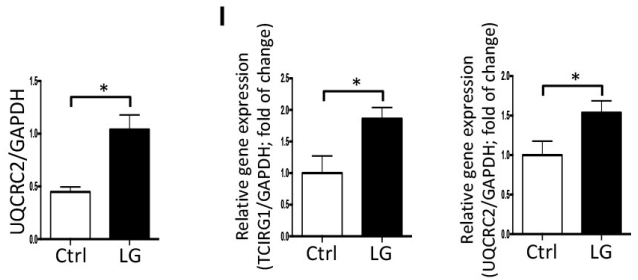

J

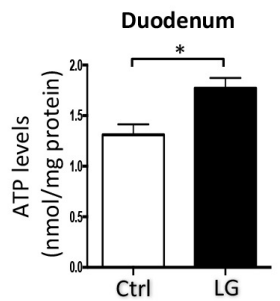

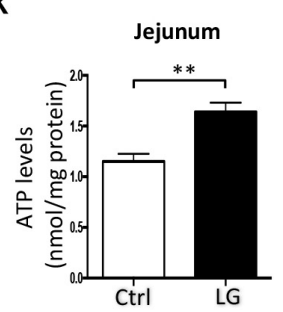

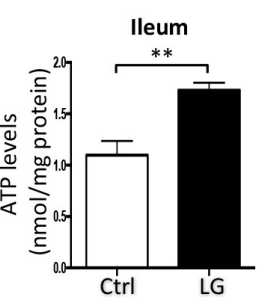

FIGURE 6 | Oral administration of L. gasseri LA39 activates OXPHOS pathway and increases ATP levels in the intestinal epithelial cells of early-weaned piglets. (A-C) Western blotting analysis for the expression levels of TCIRG1, UQCRC2, and GAPDH in the duodenal epithelial cells (A). Normalization and quantitation of TCIRG1/GAPDH and UQCRC2/GAPDH are shown in the corresponding bar chart (B). (C) The relative mRNA expression levels of TCIRG1, UQCRC2, and GAPDH in duodenal epithelial cells. Normalization and quantitation of TCIRG1/GAPDH and UQCRC2/GAPDH were shown in corresponding bar chart. (D-F) Western blotting analysis for the expression levels of TCIRG1, UQCRC2, and GAPDH in the jejunal epithelial cells (D). Normalization and quantitation of TCIRG1/GAPDH and UQCRC2/GAPDH are shown in the corresponding bar chart (E). (F) The relative mRNA expression levels of TCIRG1, UQCRC2, and GAPDH in jejunal epithelial cells. Normalization and quantitation of TCIRG1/GAPDH and UQCRC2/GAPDH were shown in corresponding bar chart. (G-I) Western blotting analysis for the expression levels of TCIRG1, UQCRC2, and GAPDH in the ileal epithelial cells (G). Normalization and quantitation of TCIRG1/GAPDH and UQCRC2/GAPDH are shown in the corresponding bar chart (H). (I) The relative mRNA expression levels of TCIRG1, UQCRC2, and GAPDH in ileal epithelial cells. Normalization and quantitation of TCIRG1/GAPDH and UQCRC2/GAPDH were shown in corresponding bar chart. (J-L) ATP levels of intestinal epithelial cells, including duodenum (J), jejunum (K), and ileum (L). The ATP concentration (nmol/L) was normalized to the total protein concentration (mg/L) of WCLs. Data are shown as mean \pm SEM; $n=3$ (B,E,H); $n=5$ (C,F, I, J-L). ${ }^{* *} p<0.01,{ }^{*} p<0.05$, and ns, not significant.

and ileum (Figures 6G-I), respectively]. The ATP levels in the intestinal epithelial cells were also significantly increased by oral gavage of L. gasseri LA39 (Figures 6J-L). These findings further indicate that $L$. gasseri LA39 can activate the OXPHOS pathway, resulting in an increase in the energy production in intestinal epithelial cells of weaned piglets. These findings suggest that $L$. gasseri LA39 may be used in intestinal epithelial energy production improvement in piglets upon early weaning stress. 


\section{DISCUSSION}

In this study, we reported an important role of L. gasseri LA39 in OXPHOS pathway activation in the intestinal epithelial cells of piglets. Our findings suggest that L. gasseri LA39 may be used in increasing the intestinal energy production in mammals. Increasing evidence has linked the intestinal microbiota to intestinal epithelial protein expression regulation (Olivares et al., 2012; Siciliano and Mazzeo, 2012; van de Guchte et al., 2012; Luo et al., 2013; Ayllón et al., 2017). This study presents evidence that the intestinal epithelial OXPHOS pathway is significantly activated by L. gasseri LA39 and facilitates our understanding about the regulatory role of the intestinal microbes in the intestinal epithelium.

The present study used a canonical in vitro model of intestinal microbial interaction with host epithelium to evaluate the regulatory role of $L$. gasseri LA39 on the protein expression profiles in the porcine intestinal epithelial cell. Such an in vitro model of the intestinal epithelium-microbe interaction has been regarded as an efficient approach that has several advantages, including direct-contact co-cultures, simplicity, and convenience (Parlesak et al., 2004; van Nuenen et al., 2005). However, the intestinal epithelium-microbial co-culture approach may be limited in some experiments involving strict anaerobic microbes, which are unable to survive under aerobic conditions (Kim et al., 2012, 2016). The L. gasseri LA39 used in the present study is a facultative anaerobe and can also survive under aerobic conditions; thereby such an in vitro model can be appropriately used to investigate the intestinal epithelial responses to $L$. gasseri LA39. Our data revealed a significant alteration in the protein expression profiles of the intestinal epithelium by L. gasseri LA39 treatment, further suggesting the efficiency of this in vitro model in determining the intestinal epithelium-microbe interaction.

This study used the iTRAQ strategy to investigate the intestinal epithelial responses to L. gasseri LA39. Many studies have used proteomics to evaluate the intestinal epitheliummicrobe interaction (Olivares et al., 2012; Siciliano and Mazzeo, 2012; van de Guchte et al., 2012; Luo et al., 2013; Ayllón et al., 2017). However, due to the limited identification capacity of traditional 2-DE-based proteomics strategies (Sun et al., 2012), investigating the integral effect of intestinal microbes on intestinal epithelium using a more advanced method will be of great significance. Recently, iTRAQ has been widely used to investigate the alteration of protein expression profiles due to its several advantages, including high sensitivity, high-throughput, and accuracy (Treumann and Thiede, 2010). Our previous study has revealed that leucine supplementation in diet induces an energy metabolism switch from OXPHOS toward glycolysis in intestinal epithelial cells of the weaned piglets, using iTRAQ strategy (Hu et al., 2017). However, the iTRAQ strategy has not been used to investigate the effects of microbes on the intestinal epithelium. Thus, the present study provides a new idea about the method for the intestinal epithelium-microbes interaction research in the future.

Our data revealed that $L$. gasseri LA39 can activate the OXPHOS pathway and increase the energy production in the intestinal epithelium of piglets both in vitro and in vivo. ATP, the major cellular energy source, is generated by both OXPHOS pathway and glycolysis pathway (Vander Heiden et al., 2009). Importantly, the OXPHOS pathway shows the maximum efficacy for producing the cellular ATP. According to the OXPHOS pathways, UQCRC2 protein belongs to the complex $\mathrm{V}$ in respiratory chain. It's known to us that complex $\mathrm{V}$ has the function in producing the gradient of protons (or hydrogen ions) between two sides of the inner mitochondrial membrane, which is the essential basis for ATP production (Saada, 2014). According to the OXPHOS pathways, the TCIRG1 protein belongs to the complex $\mathrm{V}$ in respiratory chain. It's known to us that complex $\mathrm{V}$ has the function in producing ATP (Saada, 2014). Thus, it's reasonable that the L. gasseri LA39-mediated up-regulations in TCIRG1 and UQCRC2 proteins expression justify the increase in ATP levels. To our knowledge, there is no report about the regulation of the intestinal epithelial OXPHOS pathway by the intestinal microbes, though our previous study has revealed that dietary leucine supplementation induces an energy metabolism switch from OXPHOS toward glycolysis in the intestinal epithelial cells of the weaned piglets (Hu et al., 2017). Thus, for the first time, our data indicated that L. gasseri LA39 activates the OXPHOS pathway and increase the energy production in the intestinal epithelium of piglets, further suggesting an important role of the intestinal epithelium-microbe interaction on host health. Early weaning strategy, which facilitates the shortening of the cycle of slaughter in piglets and improves the reproductive performance in sows, has been widely used in pig production (Campbell et al., 2013). However, early weaning strategy induced a transformation in the diet from liquid milk toward solid feed, which causes a reduction in feed intake and intestinal indigestion in early-weaned piglets (Lalles et al., 2007). These adverse factors will cause an insufficient energy supply, thereby decreasing the growth and increasing the intestinal damages in early-weaned piglets. Thus, L. gasseri LA39 may be a probiotic candidate functioning in intestinal energy production promotion in earlyweaned piglets. These results also suggest that L. gasseri LA39 may contribute to the intestinal energy production in human beings (especially infants) because of the similarities in the anatomy and nutritional physiology between pigs (Sus scrofa) and human beings (Vodicka et al., 2005; Maxmen, 2012; Meurens et al., 2012; Nielsen et al., 2014). The results also indicated that several KEGG pathways, including complement and coagulation cascades and NF-kappa B signaling pathway were enriched. Importantly, the expression levels of the differentially expressed proteins prothrombin, urokinase-type plasminogen activator, and vitronectin, involved in the complement and coagulation cascades pathway, significantly increased with L. gasseri LA39 treatment, suggesting the activation of the intestinal immune defense system. The expression levels of two differentially expressed proteins interleukin-8 and urokinasetype plasminogen activator, involved in the NF-kappa B signaling pathway, also significantly increased with L. gasseri LA39 treatment, indicating that the NF-kappa B signaling pathway in the intestinal epithelial cells was activated by L. gasseri LA39. These findings further suggest that the regulatory role of L. gasseri LA39 in intestinal epithelial cells is of complexity and many 
biological events need to be further explored, though we mainly focused on the OXPHOS pathway.

\section{CONCLUSION}

In conclusion, our data revealed that L. gasseri LA39 activates the OXPHOS pathway and significantly increases the ATP levels in the porcine intestinal epithelial cells in vitro and in vivo. These findings suggest an important role of L. gasseri LA39 in increasing intestinal the energy production and the interactions of gut microbes with host physiology in mammals.

\section{AUTHOR CONTRIBUTIONS}

JH, LM, and XY designed the study. JH, LM, WZ, and YN conducted the experiments. JH, LM, and XY wrote the paper with

\section{REFERENCES}

Ayllón, N., Jimenez-Marin, A., Arguello, H., Zaldivar-Lopez, S., Villar, M., Aguilar, C., et al. (2017). Comparative proteomics reveals differences in hostpathogen interaction between infectious and commensal relationship with Campylobacter jejuni. Front. Cell. Infect. Microbiol. 7:145. doi: 10.3389/fcimb. 2017.00145

Backhed, F., Manchester, J. K., Semenkovich, C. F., and Gordon, J. I. (2007). Mechanisms underlying the resistance to diet-induced obesity in germ-free mice. Proc. Natl. Acad. Sci. U.S.A. 104, 979-984. doi: 10.1073/pnas.06053 74104

Borody, T. J., and Khoruts, A. (2011). Fecal microbiota transplantation and emerging applications. Nat. Rev. Gastroenterol. Hepatol. 9, 88-96. doi: 10.1038/ nrgastro.2011.244

Campbell, J. M., Crenshaw, J. D., and Polo, J. (2013). The biological stress of early weaned piglets. J. Anim. Sci. Biotechnol. 4:19. doi: 10.1186/2049-1891-4-19

Fan, Q., Long, B., Yan, G., Wang, Z., Shi, M., Bao, X., et al. (2017). Dietary leucine supplementation alters energy metabolism and induces slow-to-fast transitions in longissimus dorsi muscle of weanling piglets. Br. J. Nutr. 117, 1222-1234. doi: $10.1017 /$ s0007114517001209

Hill, C., Guarner, F., Reid, G., Gibson, G. R., Merenstein, D. J., Pot, B., et al. (2014). Expert consensus document. The International Scientific Association for Probiotics and Prebiotics consensus statement on the scope and appropriate use of the term probiotic. Nat. Rev. Gastroenterol. Hepatol. 11, 506-514. doi: 10.1038/nrgastro.2014.66

Hu, J., Chen, L., Zheng, W., Shi, M., Liu, L., Xie, C., et al. (2018). Lactobacillus frumenti facilitates intestinal epithelial barrier function maintenance in earlyweaned piglets. Front. Microbiol. 9:897. doi: 10.3389/fmicb.2018.00897

Hu, Z., Du, M., Lai, W., Liang, Y., Liu, Q., Mo, Y., et al. (2018). Energy metabolism in the bone is associated with histomorphometric changes in rats with hyperthyroidism. Cell. Physiol. Biochem. 46, 1471-1482. doi: 10.1159/ 000489187

Hu, J., Nie, Y., Chen, J., Zhang, Y., Wang, Z., Fan, Q., et al. (2016). Gradual changes of gut microbiota in weaned miniature piglets. Front. Microbiol. 7:1727. doi: $10.3389 /$ fmicb.2016.01727

Hu, J., Nie, Y., Chen, S., Xie, C., Fan, Q., Wang, Z., et al. (2017). Leucine reduces reactive oxygen species levels via an energy metabolism switch by activation of the mTOR-HIF-1alpha pathway in porcine intestinal epithelial cells. Int. J. Biochem. Cell. Biol. 89, 42-56. doi: 10.1016/j.biocel.2017.05.026

Huang, H. Y., Zhang, W. T., Jiang, W. Y., Chen, S. Z., Liu, Y., Ge, X., et al. (2015). RhoGDIbeta inhibits bone morphogenetic protein 4 (BMP4)induced adipocyte lineage commitment and favors smooth muscle-like cell differentiation. J. Biol. Chem. 290, 11119-11129. doi: 10.1074/jbc.M114.608075 the help of all the authors. All authors read and approved the final version of the manuscript.

\section{FUNDING}

This work was supported by the National Natural Science Foundation of China (31730090, 31520103915, and 31322053), the National Key Research and Development Program of China (2017YFD0500503 and 2018YFD0500404), Hubei Provincial Natural Science Foundation of China (2018CFA020), and the Fundamental Research Funds for the Central Universities (2013PY056, 2662015PY111, and 2013JQ001).

\section{ACKNOWLEDGMENTS}

We thank all present and past members of Yan Laboratory who have contributed their comments and ideas.

Huang, Y., Liao, M., Yang, Q., Xiao, J., Hu, Z., and Cao, H. (2018). iTRAQbased quantitative proteome revealed metabolic changes of Sitophilus zeamais in response to terpinen-4-ol fumigation. Pest Manag. Sci. doi: 10.1002/ps.5135 [Epub ahead of print]. doi: 10.1002/ps.5135

Ivanov, I. I., Atarashi, K., Manel, N., Brodie, E. L., Shima, T., Karaoz, U., et al. (2009). Induction of intestinal Th17 cells by segmented filamentous bacteria. Cell 139, 485-498. doi: 10.1016/j.cell.2009.09.033

Kawai, Y., Ishii, Y., Uemura, K., Kitazawa, H., Saito, T., and Itoh, T. (2001). Lactobacillus reuteri LA6 and Lactobacillus gasseri LA39 isolated from faeces of the same human infant produce identical cyclic bacteriocin. Food Microbiol. 18, 407-415. doi: 10.1006/fmic.2001.0412

Kawai, Y., Saito, T., Toba, T., Samant, S. K., and Itoh, T. (1994). Isolation and characterization of a highly hydrophobic new bacteriocin (gassericin A) from Lactobacillus gasseri LA39. Biosci. Biotechnol. Biochem. 58, 1218-1221. doi: 10.1271/bbb.58.1218

Kim, H. J., Huh, D., Hamilton, G., and Ingber, D. E. (2012). Human gut-on-a-chip inhabited by microbial flora that experiences intestinal peristalsis-like motions and flow. Lab Chip 12, 2165-2174. doi: 10.1039/c2lc40074j

Kim, H. J., Li, H., Collins, J. J., and Ingber, D. E. (2016). Contributions of microbiome and mechanical deformation to intestinal bacterial overgrowth and inflammation in a human gut-on-a-chip. Proc. Natl. Acad. Sci. U.S.A. 113, E7-E15. doi: 10.1073/pnas.1522193112

Kim, M., Ashida, H., Ogawa, M., Yoshikawa, Y., Mimuro, H., and Sasakawa, C. (2010). Bacterial interactions with the host epithelium. Cell Host Microbe 8, 20-35. doi: 10.1016/j.chom.2010.06.006

Kleerebezem, M., Hols, P., Bernard, E., Rolain, T., Zhou, M., Siezen, R. J., et al. (2010). The extracellular biology of the lactobacilli. FEMS. Microbiol. Rev. 34, 199-230. doi: 10.1111/j.1574-6976.2010.00208.x

Lalles, J. P., Bosi, P., Smidt, H., and Stokes, C. R. (2007). Nutritional management of gut health in pigs around weaning. Proc. Nutr. Soc. 66, 260-268. doi: 10.1017/ s0029665107005484

Lemon, K. P., Armitage, G. C., Relman, D. A., and Fischbach, M. A. (2012). Microbiota-targeted therapies: an ecological perspective. Sci. Transl. Med. 4:137rv135. doi: 10.1126/scitranslmed.3004183

Li, M., Hui, X., Pan, Y., Cai, H., Dai, Y., and Xu, X. (2018). Proteomic evaluation of human umbilical cord tissue exposed to polybrominated diphenyl ethers in an e-waste recycling area. Environ. Int. 111, 362-371. doi: 10.1016/j.envint.2017. 09.016

Long, B., Yin, C., Fan, Q., Yan, G., Wang, Z., Li, X., et al. (2016). Global liver proteome analysis using iTRAQ reveals AMPK-mTOR-autophagy signaling is altered by intrauterine growth restriction in newborn piglets. J. Proteome Res. 15, 1262-1273. doi: 10.1021/acs.jproteome. 6b00001 
Luo, J., Zheng, A., Meng, K., Chang, W., Bai, Y., Li, K., et al. (2013). Proteome changes in the intestinal mucosa of broiler (Gallus gallus) activated by probiotic Enterococcus faecium. J. Proteomics 91, 226-241. doi: 10.1016/j.jprot.2013. 07.017

Maxmen, A. (2012). Model pigs face messy path. Nature 486:453. doi: 10.1038/ $486453 \mathrm{a}$

Meurens, F., Summerfield, A., Nauwynck, H., Saif, L., and Gerdts, V. (2012). The pig: a model for human infectious diseases. Trends Microbiol. 20, 50-57. doi: 10.1016/j.tim.2011.11.002

Nielsen, K. L., Hartvigsen, M. L., Hedemann, M. S., Laerke, H. N., Hermansen, K., and Bach Knudsen, K. E. (2014). Similar metabolic responses in pigs and humans to breads with different contents and compositions of dietary fibers: a metabolomics study. Am. J. Clin. Nutr. 99, 941-949. doi: 10.3945/ajcn.113. 074724

Olivares, M., Laparra, M., and Sanz, Y. (2012). Oral administration of Bifidobacterium longum CECT 7347 modulates jejunal proteome in an in vivo gliadin-induced enteropathy animal model. J. Proteomics 77, 310-320. doi: 10.1016/j.jprot.2012.09.005

Parlesak, A., Haller, D., Brinz, S., Baeuerlein, A., and Bode, C. (2004). Modulation of cytokine release by differentiated CACO-2 cells in a compartmentalized coculture model with mononuclear leucocytes and nonpathogenic bacteria. Scand. J. Immunol. 60, 477-485. doi: 10.1111/j.0300-9475.2004.01495.x

Ren, Y., Hao, P., Dutta, B., Cheow, E. S., Sim, K. H., Gan, C. S., et al. (2013). Hypoxia modulates A431 cellular pathways association to tumor radioresistance and enhanced migration revealed by comprehensive proteomic and functional studies. Mol. Cell. Proteomics 12, 485-498. doi: 10.1074/mcp.M112. 018325

Rhein, V., Song, X., Wiesner, A., Ittner, L. M., Baysang, G., Meier, F., et al. (2009). Amyloid-beta and tau synergistically impair the oxidative phosphorylation system in triple transgenic Alzheimer's disease mice. Proc. Natl. Acad. Sci. U.S.A. 106, 20057-20062. doi: 10.1073/pnas.0905529106

Saada, A. (2014). Mitochondria: mitochondrial OXPHOS (dys) function ex vivothe use of primary fibroblasts. Int. J. Biochem. Cell. Biol. 48, 60-65. doi: 10.1016/ j.biocel.2013.12.010

Savitski, M., Wilhelm, M., Hahne, H., Kuster, B., and Bantscheff, M. (2015). A scalable approach for protein false discovery rate estimation in large proteomic data sets. Mol. Cell. Proteomics 14, 2394-2404. doi: 10.1074/mcp. M114.046995

Shi, Q., Chen, L. N., Zhang, B. Y., Xiao, K., Zhou, W., Chen, C., et al. (2015). Proteomics analyses for the global proteins in the brain tissues of different human prion diseases. Mol. Cell. Proteomics 14, 854-869. doi: 10.1074/mcp. M114.038018

Siciliano, R. A., and Mazzeo, M. F. (2012). Molecular mechanisms of probiotic action: a proteomic perspective. Curr. Opin. Microbiol. 15, 390-396. doi: 10. 1016/j.mib.2012.03.006

Sommer, F., and Backhed, F. (2013). The gut microbiota-masters of host development and physiology. Nat. Rev. Microbiol. 11, 227-238. doi: 10.1038/ nrmicro2974

Sun, H., Li, M., Gong, L., Liu, M., Ding, F., and Gu, X. (2012). iTRAQ-coupled 2D LC-MS/MS analysis on differentially expressed proteins in denervated tibialis anterior muscle of Rattus norvegicus. Mol. Cell. Biochem. 364, 193-207. doi: 10.1007/s11010-011-1218-2

Treumann, A., and Thiede, B. (2010). Isobaric protein and peptide quantification: perspectives and issues. Expert Rev. Proteomics 7, 647-653. doi: 10.1586/epr. 10.29

van Baarlen, P., Wells, J. M., and Kleerebezem, M. (2013). Regulation of intestinal homeostasis and immunity with probiotic lactobacilli. Trends Immunol. 34, 208-215. doi: 10.1016/j.it.2013.01.005

van de Guchte, M., Chaze, T., Jan, G., and Mistou, M. Y. (2012). Properties of probiotic bacteria explored by proteomic approaches. Curr. Opin. Microbiol. 15, 381-389. doi: 10.1016/j.mib.2012.04.003

van Nuenen, M. H., de Ligt, R. A., Doornbos, R. P., van der Woude, J. C., Kuipers, E. J., and Venema, K. (2005). The influence of microbial metabolites on human intestinal epithelial cells and macrophages in vitro. FEMS. Immunol. Med. Microbiol. 45, 183-189. doi: 10.1016/j.femsim.2005.03.010

Vander Heiden, M., Cantley, L., and Thompson, C. (2009). Understanding the Warburg effect: the metabolic requirements of cell proliferation. Science 324, 1029-1033. doi: 10.1126/science.1160809

Ventura, M., O’Flaherty, S., Claesson, M. J., Turroni, F., Klaenhammer, T. R., van Sinderen, D., et al. (2009). Genome-scale analyses of health-promoting bacteria: probiogenomics. Nat. Rev. Microbiol. 7, 61-71. doi: 10.1038/nrmicro2047

Vodicka, P., Smetana, K. Jr., Dvoránková, B., Emerick, T., Xu, Y. Z., Ourednik, J., et al. (2005). The miniature pig as an animal model in biomedical research. Ann. N. Y. Acad. Sci. 1049, 161-171. doi: 10.1196/annals.1334.015

Wang, H., Wei, H., Tang, L., Lu, J., Mu, C., and Wang, C. (2018). A proteomics of gills approach to understanding salinity adaptation of Scylla paramamosain. Gene 677, 119-131. doi: 10.1016/j.gene.2018.07.059

Weatherly, D., Atwood, J., Minning, T., Cavola, C., Tarleton, R., and Orlando, R. (2005). A Heuristic method for assigning a false-discovery rate for protein identifications from Mascot database search results. Mol. Cell. Proteomics 4, 762-772. doi: 10.1074/mcp.M400215-MCP200

Wen, B., Zhou, R., Feng, Q., Wang, Q., Wang, J., and Liu, S. (2014). IQuant: an automated pipeline for quantitative proteomics based upon isobaric tags. Proteomics 14, 2280-2285. doi: 10.1002/pmic.201300361

Zhu, M., Dai, S., McClung, S., Yan, X., and Chen, S. (2009). Functional differentiation of Brassica napus guard cells and mesophyll cells revealed by comparative proteomics. Mol. Cell. Proteomics 8, 752-766. doi: 10.1074/mcp. M800343-MCP200

Conflict of Interest Statement: The authors declare that the research was conducted in the absence of any commercial or financial relationships that could be construed as a potential conflict of interest.

Copyright (c) $2018 \mathrm{Hu}, \mathrm{Ma}$, Zheng, Nie and Yan. This is an open-access article distributed under the terms of the Creative Commons Attribution License (CC BY). The use, distribution or reproduction in other forums is permitted, provided the original author(s) and the copyright owner(s) are credited and that the original publication in this journal is cited, in accordance with accepted academic practice. No use, distribution or reproduction is permitted which does not comply with these terms. 